Supporting Information

\title{
Epitope Mapping for a Pre-Clinical Bevacizumab (Avastin) Biosimilar on an Extended Construct of Vascular Endothelial Growth Factor-A using Millisecond H/D Exchange Mass Spectrometry
}

\author{
Kerene A. Brown ${ }^{1,2}$, Cristina Lento ${ }^{1,2}$, Shanthi Rajendran ${ }^{3}$, Jason Dowd ${ }^{4}$ and Derek J. Wilson ${ }^{1}{ }^{2 *}$ \\ ${ }^{1}$ Chemistry Department, York University, 4700 Keele Street, Toronto, ON, M3J 1P3, Canada \\ ${ }^{2}$ The Centre for Research in Mass Spectrometry, York University, Toronto, ON, M3J1P3, Canada \\ ${ }^{3}$ Apobiologix (division of Apotex Inc.), 4100 Weston Road, Toronto, ON M9L 2Y6, Canada \\ ${ }^{4}$ Centre for Commercialization of Regenrative Medicine, 661 University Avenue, Suite 1002, Toronto, \\ ON, M5G $1 \mathrm{M} 1$ \\ *Email: dkwilson@yorku.ca
}




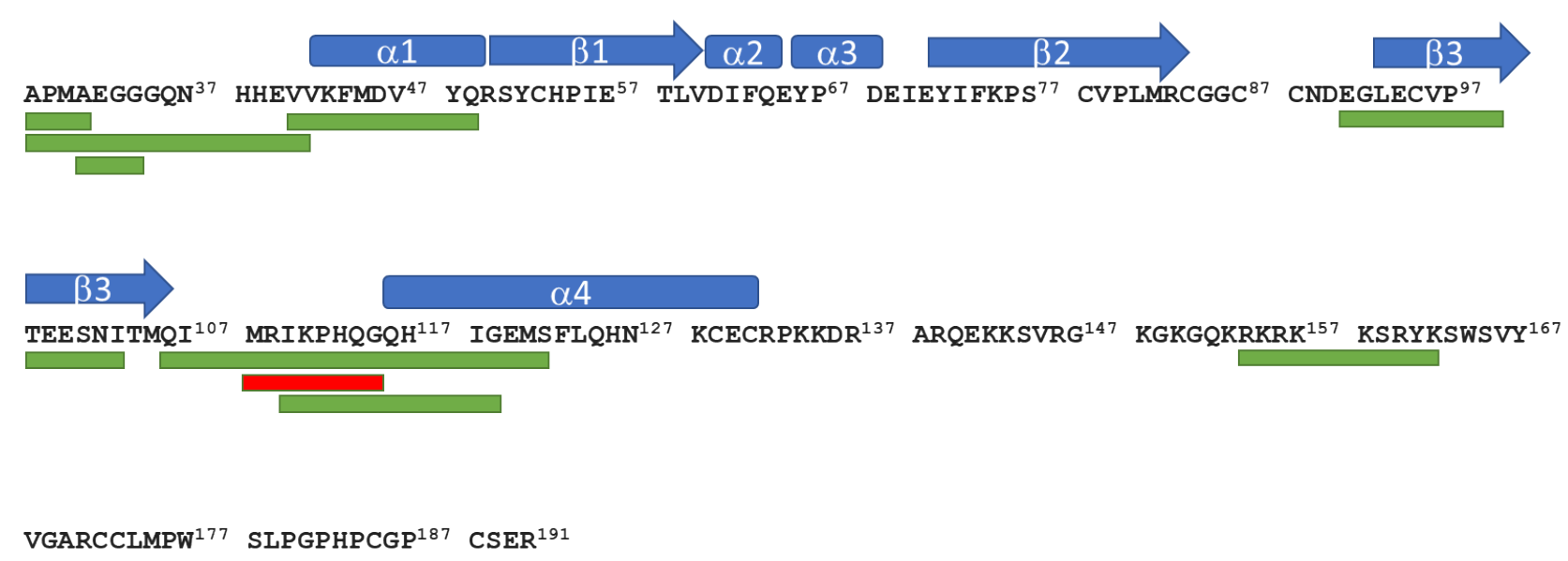

Figure S1. A schematic depiction of secondary structure for the VEGF- $\mathrm{A}_{165}$ construct used in this study. The numbering system corresponds to the full-length VEGF-A sequence. Known secondary structures are shown above the sequence (blue) while peptides used in the analysis are shown below the sequence (green) with the key epitope peptide shown in red. 


\section{Materials and Methods}

\section{Materials}

Bevacizumab (Avastin, $149.2 \mathrm{kDa}$ ) the candidate biosimilars (149.4 kDa) were provided by Apobiologix. Recombinant Human Vascular Endothelial Growth Factor 165 (VEGF-165) was purchased form R and D systems. Deuterium oxide $\left(\mathrm{D}_{2} \mathrm{O}, 99.9 \%, 151882\right)$ and high purity acetic acid $(>99.7 \%, 695092)$ were purchased from Sigma-Aldrich (St. Louis, MO). HPLC-grade ammonium acetate and water were purchased from Fisher Chemical (Fair Lawn, NJ).

\section{Microfluidic chip fabrication}

The microfluidic chip was constructed as previously described. Briefly, a proteolytic chamber and capillary channels were engraved onto a poly methyl methacrylate (PMMA) substrate (dimensions: 0.83" x 2" x 0.25 ") and welded together with another PMMA substrate of the same dimensions. A $10 \mathrm{~cm}$ glass capillary with an outer diameter of $151 \mu \mathrm{m}$ and a $10 \mathrm{~cm} 33$ gauge stainless steel metal capillary screwed into the chip via double-winged nuts and were used as the input and output channel respectively.

The TRESI mixer was made by inserting a glass capillary (Outer Diameter $=151 \mu \mathrm{m})$ inside a metal capillary (inner diameter $=177.8 \mu \mathrm{M}$ ) creating an inter capillary space of $26 \mu \mathrm{m}$. The end of the glass capillary was sealed and a notch was cut $2 \mathrm{~mm}$ from the sealed end with the use of a laser. A three-way mixing device was used to connect the TRESI mixer, the acid line and the capillary leading to the microfluidic chip. The device was placed at the front end of a modified NanoSpray platform on a quadrupole-ion mobility-time-of-fight mass spectrometer instrument (Synapt G2-S HDMS, Waters, Milford, MA). The output channel of the microfluidic device also served a the electrospray ionization probe.

\section{Time-Resolved ElectroSpray Ionization Hydrogen Deuterium eXchange (TRESI-HDX)}

The HDX reaction occurred within the TRESI mixer in which gas tight Hamilton syringes were used to transport the reagents through the capillaries using Syringe infusion pumps (Harvard Apparatus). For equilibrium studies, $2 \mu \mathrm{M}$ VEGF-165 (or Antibody/VEGF complex) in $150 \mathrm{mM} \mathrm{C}_{2} \mathrm{H}_{3} \mathrm{O}_{2} \mathrm{NH}_{4}, \mathrm{pH} 6.7$ 
flowed through the inner capillary while deuterium flowed through the outer capillary at $2 \mu \mathrm{L} / \mathrm{min}$. For 'Kinetic' studies, $2 \mu \mathrm{M}$ VEGF in $150 \mathrm{mM} \mathrm{C}_{2} \mathrm{H}_{3} \mathrm{O}_{2} \mathrm{NH}_{4}$, $\mathrm{pH} 6.7$ flowed through the inner capillary and $2 \mu \mathrm{M}$ bevacizumab in $99.9 \%$ deuterium flowed through the outer capillary. The HDX reaction times were achieved by increasing the space between the end of the inner capillary and the outer capillary by $2,5,10$ and $20 \mathrm{~mm}$. The instrument operated with a capillary voltage of $2.5 \mathrm{kV}$ in positive ion mode with a sampling cone voltage of $20 \mathrm{~V}$. The samples were scanned over a range of 400 to $1500 \mathrm{~m} / \mathrm{z}$.

A digestion profile was acquired prior to the HDX reaction and tandem MS was then used to determine the sequence of each peptide. Data Analysis was carried out using the MS studio software ${ }^{2}$ in which the deuterium levels for each peptide in free VEGF and Antibody/VEGF complex was calculated. Differences in deuterium uptake that fell with 2 sigma (95\% confidence interval) was considered to be significant. The significant differences were then mapped onto the X-ray co-crystal. 